\title{
Sistema Mayordomo: la puerta de entrada de nuestros mayores a las nuevas tecnologías
}

\author{
Ernestina Etchemendy \\ CIBER Fisiopatología, Obesidad y Nutrición. CB06/03 ISC-III, España
}

Departamento de Personalidad, Evaluación y Ttos. Psicológicos. Universitat de València, España

ernestina@labpsitec.es

Diana Castilla

CIBER Fisiopatología, Obesidad y Nutrición. CB06/03 ISC-III, España

Departamento de Psicología Básica, Clínica y Psicobiología. Universitat Jaume I, España

Rosa María Baños

CIBER Fisiopatología, Obesidad y Nutrición. CB06/03 ISC-III. España

Departamento de Personalidad, Evaluación y Ttos. Psicológicos. Universitat de València, España

Cristina Botella

CIBER Fisiopatología, Obesidad y Nutrición. CB06/03 ISC-III, España

Departamento de Psicología Básica, Clínica y Psicobiología. Universitat Jaume I, España

\begin{abstract}
The population distribution has changed and is set around an increasingly aging population. This population change coincides with the technological revolution taking place in the XxI century, which generates a digital divide that separates old people and empower feelings of isolation, loneliness and sadness. Our team has developed the Mayordomo System. Based on the principles of positive psychology, it's main objective is to build a bridge between the technological reality of today and an older population that will continue to increase. The mayordomo System, through the use of new technologies, aims to facilitate the construction of protective psychological and social elements of a healthy quality of life. The system has been installed on two Senior Colleges of Valencia (Universitat de València and Universitat Jaume I), a nursing home and a day centre. The aim of this paper is to describe the experience of two users who used our system and analyze the effectiveness of the use of Mayordomo System upon their mood and satisfaction levels. The results show that users increased their mood and levels of satisfaction. Butler is a system that can contribute to improve the welfare of older people by facilitating the construction of protective psychological elements, such as increasing their social network and the development of leisure activities.
\end{abstract}

Keywords: elderly people, new technologies, positive psychology, successful aging

\section{Resumen}

La distribución demográfica ha cambiado y se configura alrededor de una población cada vez más envejecida. Este cambio poblacional coincide con la revolución tecnológica que se está produciendo en el siglo XxI, la cual genera una brecha digital que separa a las personas mayores y potencia sentimientos de aislamiento, soledad y tristeza. Nuestro equipo ha desarrollado el sistema Mayordomo. Basado en los principios de la psicología positiva, tiene como principal objetivo construir un puente entre la realidad tecnológica de hoy y una población mayor que seguirá en aumento. Mayordomo, a través del uso de las nuevas tecnologías, se dirige a facilitar la construcción de elementos psicológicos 
y sociales protectores de una calidad de vida saludable. El sistema Mayordomo ha sido instalado en dos universidades de mayores de la Comunidad Valenciana (Universitat de València y Universitat Jaume I), una residencia y un centro de día. El objetivo de este trabajo es describir la experiencia de dos usuarios que utilizaron nuestro sistema y analizar la eficacia de su uso sobre el estado de ánimo y los niveles de satisfacción obtenidos. Los resultados muestran que los usuarios incrementaron su estado de ánimo y niveles de satisfacción. Mayordomo es un sistema que puede contribuir a la mejora del bienestar de las personas mayores, facilitando la construcción de elementos psicológicos protectores, como es el incremento de su red social y el desarrollo de actividades placenteras.

Palabras clave: tercera edad, nuevas tecnologías, psicología positiva, envejecimiento exitoso

\section{Introducción}

La distribución demográfica ha cambiado y se configura alrededor de una población cada vez más envejecida. Por otro lado, esta realidad sociodemográfica se entrecruza con la revolución tecnológica iniciada a finales del siglo $\mathrm{xx}$, la cual ha redefinido la forma de funcionamiento de las relaciones humanas. El mundo ha cambiado y lo ha hecho a una velocidad vertiginosa. Este desfase generacional puede traer aparejado que la persona mayor se sienta descolocada y ajena a este nuevo mundo. El diálogo con sus hijos, con sus nietos, las fotos, la música, el diario, etcétera, está cada vez más centralizado en un cambiante formato digital. Esta desconexión potencia la vulnerabilidad de esta población a sufrir del estado de ánimo, problemas de ansiedad, etc. Por contrapartida, numerosos estudios científicos indican lo beneficioso que resulta acercar las nuevas tecnologías de la información y la comunicación (TICs) a las personas de la tercera edad, convirtiéndose en una potente herramienta de comunicación, ocio, socialización y entretenimiento (Blit-Cohen y Litwin, 2004; Marek van de Catering, 2005; Wellman y Frank, 2001)

Frente a esta realidad de encuentros y desencuentros surge el sistema Mayordomo. Basado en los principios de la psicología positiva y con el objetivo de construir un puente entre la realidad tecnológica de hoy y una población mayor que seguirá en aumento, se dirige a facilitar la construcción de elementos psicológicos y sociales protectores de una calidad de vida saludable.
El presente trabajo se dirige a presentar una breve descripción del sistema Mayordomo, la experiencia de dos usuarias que utilizaron nuestro sistema, y analizar la eficacia de su uso sobre el estado de ánimo y los niveles de satisfacción obtenidos.

\subsection{Proyecto Mayordomo: descripción del sistema}

El sistema engloba una plataforma tecnológica multiusuario, que usa Internet como red de enlace y que incluye distintas líneas de intervención. Actúa a un nivel de intervención primaria, facilitando y optimizando la labor del profesional, además de mejorar la calidad de vida de la población mayor. Los beneficios del sistema se encuentran a tres niveles:

1. A nivel diagnóstico, su objetivo es la detección de sintomatología ansiosa y depresiva. Finalizada la evaluación el sistema de forma inteligente decide cuales son las opciones terapéuticas y lúdicas recomendadas. Si detecta que el estado de ánimo está alterado, el sistema envía un aviso al profesional responsable. Además, la aplicación resume toda la información que obtiene para que el profesional pueda acceder a ella fácilmente y obtener análisis de períodos, gráficos y tabla de datos.

2. A nivel clínico-terapéutico cuenta con dos recursos:

- Mundos virtuales para generar emociones positivas. Esta herramienta incluye dos entornos virtuales en $3 \mathrm{D}$ que presentan al usuario distintos estímulos visuales y audi- 
tivos con el objetivo de producir cambios en su estado de ánimo (alegría y relajación).

- Libro de la vida terapéutico. La herramienta es administrada por el profesional y se trata de un programa de entrenamiento en memoria autobiográfica positiva.

3. Por último, el nivel lúdico-recreativo, dirigido a incrementar las relaciones sociales, fomentar distintas habilidades en comunicación, compartir la memoria vital y aprender nuevas tecnologías. Incluye correo electrónico, búsqueda de amigos en la red social Mayordomo, Libro de la vida (blog), fácil acceso a Internet y biblioteca personalizada de fotos y melodías. El sistema también permite que la persona mayor pueda comunicarse con un familiar o amigo externo a la red Mayordomo y compartir con dicha persona las herramientas lúdicas.

El sistema Mayordomo ha sido instalado en dos universidades de Mayores de la Comunidad Valenciana (Universitat de València y Universitat Jaume I), una residencia y un centro de día. El objetivo de este trabajo es describir la experiencia de dos usuarias que utilizaron nuestro sistema y analizar la eficacia de su uso sobre el estado de ánimo y los niveles de satisfacción obtenidos.

\section{Método}

Las participantes fueron dos mujeres de 65 y 74 años de edad. Una de ellas (usuaria A) asistía a la Universidad de Mayores de la Universitat Jaume I, tenía un nivel de estudios superior y estaba casada. La otra usuaria (usuaria B) concurría al centro de día, tenía un nivel de estudios básico, era viuda y vivia sola. Ninguna de ellas tenía experiencia con el uso de ordenadores. Ninguna participante presentó problemas psicológicos ni cognitivos, ni mostraron puntuaciones elevadas ni en ansiedad ni en depresión. Ambas usuarias utilizaron Mayordomo durante 10 sesiones. Cada sesión tuvo una duración aproximada de 60 minutos.

\subsection{Medidas}

Se utilizaron los siguientes instrumentos para evaluar la esfera emocional y la satisfacción con el sistema:
1. Escala de estado de animo general (EAG). Se trata de una escala analógica, diseñada específicamente para este estudio, formada por 7 expresiones faciales que van desde 1 (cara de máxima tristeza) a 7 (cara de máxima felicidad). Antes y después de utilizar el sistema las usuarias elegían la expresión facial que mejor representaba su estado de ánimo en ese momento.

2. Nivel de satisfacción (Ns). En este instrumento, las usuarias valoraban el grado de satisfacción con lo que habian hecho en Mayordomo en esa sesión. Se trata de una escala analógica visual formada por 7 expresiones faciales, ordenadas de 0 (cara de máxima insatisfacción) a 6 (cara de máxima satisfacción).

\subsection{Procedimiento}

Una vez el sistema Mayordomo fue instalado en dos universidades de mayores de la Comunidad Valenciana (Universitat de Valéncia y Universitat Jaume I), una residencia y un centro de día, nos pusimos en contacto con personas mayores de las tres instituciones y se les invitó a participar en nuestro estudio. A aquellas personas interesadas en participar se les administró la versión estado del StateTrait Anxiety Inventory Scale (sTAI-s) (Spielberger, Gorsuch y Lushene, 1970) y la escala Yesavage-15 (Sheikh y Yesavage, 1986), con el fin de detectar índices clínicos de ansiedad y depresión, los cuales se habían establecido como criterios de exclusión. Una vez firmado el consentimiento informado, se les entregaba la clave de acceso al sistema y las participantes comenzaban a usar el sistema Mayordomo una vez por semana. Antes y después de cada sesión, una investigadora les administraba la EAG. La escala de satisfacción se administraba junto con la EAG después de cada sesión.

En el caso de las universidades, una investigadora permanecía en una sala contigua para cualquier consulta que las participantes pudieran tener, aunque se alentaba a las usuarias a guiarse por las instrucciones que el sistema Mayordomo iba dando. En el caso del centro de día y la residencia, una investigadora brindaba un apoyo más directo en las primeras sesiones y posteriormente iba asumiendo 


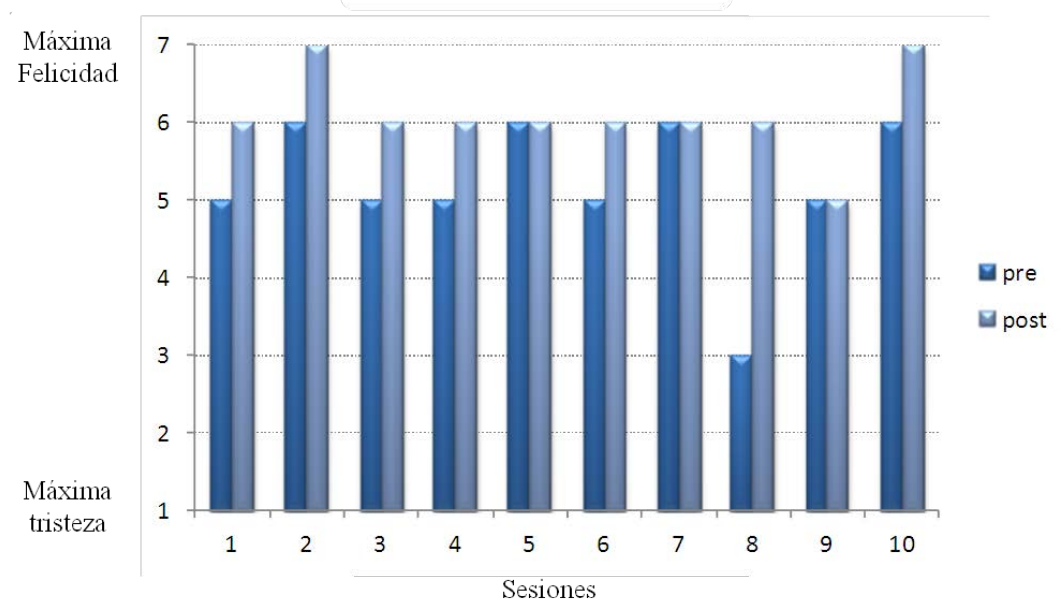

Figura 1. EAG usuaria A

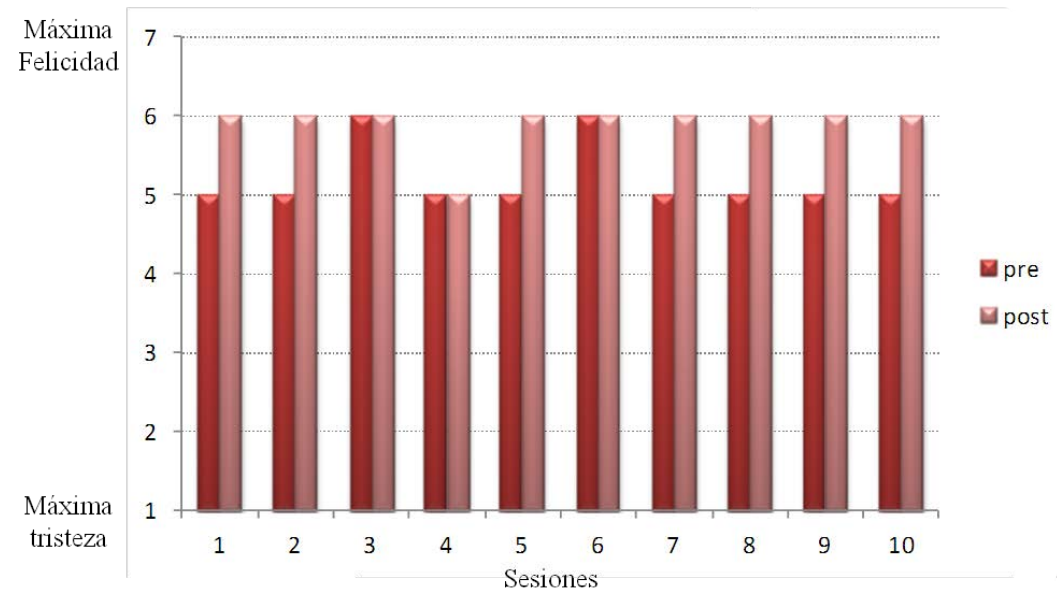

Figura 2. EAG usuaria B

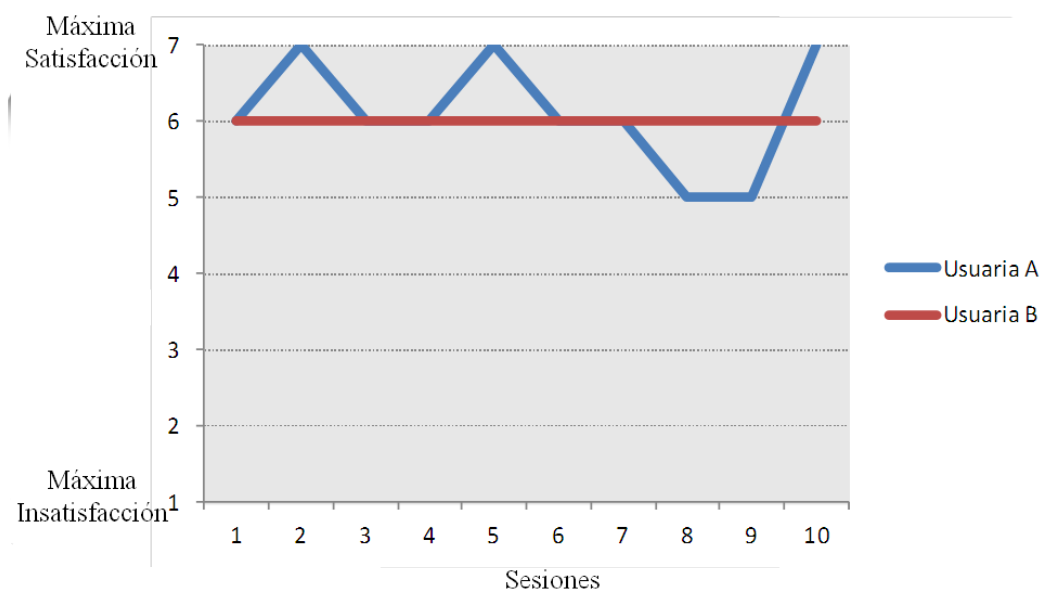

Figura 3. Ns usuaria $A$ y $B$ el mismo rol que se asumía en las universidades desde las primeras sesiones.

\section{Resultados}

Escala de estado de ánimo general (EAG)(veánse las figuras 1 y 2): los resultados muestran un incremento del Estado de Ánimo después de utilizar el sistema Mayordomo, en ambas usuarias, en la mayoría de las sesiones. En ninguna sesión se observó un descenso del EAg tras utilizar Mayordomo. Respecto a la usuaria A (véase figura 1), se observó un estado de ánimo elevado en todas las sesiones, exceptuando la sesión 8 donde se registró un EAG bajo antes de utilizar el sistema. Por otro lado, el EAG de la usuaria subió en 7 de las 10 sesiones de uso; incluso en la sesión 8 , la usuaria registró un EAG elevado tras utilizar nuestro sistema. Respecto a la usuaria B (ver figura 2), se observó un estado de ánimo elevado en todas las sesiones y un aumento del mismo en 7 de las 10 sesiones.

Nivel de Satisfacción (Ns)(váse la figura 3): ambas usuarias, en todas las sesiones, mostraron elevados niveles de satisfacción tras utilizar nuestro sistema.

A continuación, se presenta una breve descripción de las experiencias de las participantes con el uso del sistema Mayordomo (con el objetivo de mantener en el anonimato cada una de las experiencias se han omitido los nombres correspondientes):

Usuaria A: se trata de una usuaria que dedica mucho tiempo libre a escribir, sin embargo, debido a su miedo a las TIC, lo hacía con una máquina de escribir tradicional. Antes del estudio, ella expresó sentir fobia por las TIC. Ella, creía que a su edad ya no podría aprender este tipo de tecnologías. Tras 10 sesiones de uso con Mayordomo, se compró una cámara digital, un 
ordenador portátil para ampliar el tiempo de uso del ordenador y de Mayordomo y comenzó a realizar sus escritos utilizando el Word ${ }^{\text {TM }}$.

A continuación, se extraen algunos fragmentos de la entrevista final realizada por un investigador: «El método de Mayordomo es muy sencillo para los principiantes, te quita el miedo y te va dando confianza cuando descubres que conocer y practicar con un ordenador no es tan difícil como imaginas en un principio. Es como cuando aprendes a desplazarte con una bicicleta, y descubres que es más rápido y cómodo que ir a pie». «Con Mayordomo lo primero que te sucede es que pierdes el miedo, y luego quieres continuar con más. Antes tenía miedo de tocar y estropear y no me atrevía con nada. Y ahora no me da miedo teclear, buscar, probar con este botón y con el otro. Siempre tenía miedo y cada vez que voy practicando lo voy perdiendo" "Meterme en Internet, descubrir canciones de cuando éramos jóvenes, buscar un relato literario... y no sólo le he perdido el miedo, sino que ha sido una experiencia gratificante $100 \%$. Antes cuando se rompían las cosas pensaba que era mi culpa, ahora cuando se rompe algo busco el manual de instrucciones. Por ejemplo, cuando me han puesto el TDT en la TV, el primer día tuvimos que cambiar de mando y no sabíamos exactamente cómo funcionaba; la TV estaba a cero. Y fui viendo y leyendo el manual e hice la TV funcionar, $\mathrm{y}$ eso es algo que antes no me habría atrevido. Y da mucha alegría saber que puedes hacer estas cosas. La actitud hacia la tecnología es distinta, es muy positiva».

Usuaria B: Esta usuaria tiene varias hijas, una de las cuales no vive en España, por lo que el uso de la videoconferencia podía tener especial interés. Cuando comenzamos a utilizar esta herramienta, tanto la madre como la hija se mostraban un tanto inseguras con la videollamada, pero al cabo del tiempo fueron tomando confianza y hablar con su hija llegó a ser algo habitual en cada sesión. Cada mañana, su hija esperaba a su madre al otro lado del ordenador para desayunar mientras charlaban y se saludaban. Una de las herramientas que más le gustaban a esta usuaria era Internet. Le encantaba ver vídeos de sus artistas favoritos y poder disfrutar de sus canciones una y otra vez. Otra herramienta que la usuaria especialmente disfrutaba era el correo. Al principio se trataba solo de correos a sus hijas, pero también escribía a algunos de sus compañeros del centro. Había un usuario con el que tenía muy buena relación y que además se trataba de otro de los participantes de nuestro estudio. Con lo cual, la usuaria aprovechaba para escribirle comentando lo que habían hecho en el centro, preocupándose por él si no había asistido ese día, animándole, etcétera. Se estableció una dinámica de correos muy agradable, dado que el otro usuario siempre devolvía esas cartas con palabras de agradecimiento. Además, cuando había algún tipo de celebración o fiesta, la usuaria pedía las fotos y luego las enviaba a sus contactos a través del correo electrónico.

\section{Conclusión}

Diversos estudios han mostrado el impacto positivo de diferentes herramientas derivadas de las TiC, sobre la comunicación, conectividad social, esfera afectiva, física y emocional, en personas de la tercera edad (Caoutteet y otros 2007; Gowans y otros 2004; Nilsson y otros 2003; Wright, 2000). Ahora bien, estos trabajos se han centrado en un solo recurso tecnológico (videoconferencia o escuchar música o aprender a navegar por la red).

Mayordomo continúa esta misma línea, pero reuniendo en una sola plataforma varias herramientas. Se trata de un sistema especialmente adaptado a las necesidades de las personas mayores y dirigido a promover la salud, la satisfacción y el bienestar personal, utilizando para ello una serie de estrategias, como son el ejercicio de las capacidades emocionales, el aprendizaje en nuevas aptitudes de comunicación, el aumento en la red de apoyo social, la curiosidad y la satisfacción por descubrirse y sorprenderse haciendo cosas que quizá nunca había hecho antes.

Como se observó en los resultados, el uso de Mayordomo produjo un aumento en el EAG y altos niveles de satisfacción, en todas las sesiones, en ambas usuarias. Por otro lado, la información cualitativa ejemplifica lo que las escalas señalan. Ambos casos manifiestan lo satisfactorio y positivo que resultó el acercar las TIC orientadas a fomentar la conectividad social, el ocio y el entretenimiento 
a nuestras usuarias. Sin embargo, este estudio tiene claras limitaciones metodológicas que impide extrapolar sus resultados. La muestra está configurada por tan solo 2 usuarias, las cuales no presentan ningún deterioro cognitivo o afectivo. Es necesario probar el sistema en una muestra de sujetos más amplia, con presencia de sintomatología ansiosa y depresiva y con un mayor número se sesiones. De esta manera, podríamos observar si los beneficios observados en el presente estudio se extienden a una mayor cantidad de usuarios y si los efectos generados por el sistema se mantienen, potencian o disminuyen con el paso de tiempo.

\section{Referencias}

Blit-Cohen, E., y Litwin, H. (2004). Elder participation in cyberspace: A qualitative analysis of Israeli retirees. Journal of Aging Studies, 18(4), 385-398.

Caoutte, A., Vincent, C., y Montreuli, B. (2007). Use of telemonitoring by elders at home: actual practice and potential. Canadian Journal of Occupational Therapy, 74(5), 382-392.

Gowans, G., Campbell, J., Alm, N., Astell, A., Ellis, M. \& Dye, R. (2004). Designing a Multimedia Conversation Aid for Reminiscence Therapy in Dementia Care Environments. En Proceeding CHI '03 extended abstracts on Human factors in computing systems (pp. 825-836). Viena: ACM Press.

Nilsson, M., Johansson, S., y Hakansson, M. (2003). Nostalgia: An Evocative Tangible Interface for Elderly Users. En Proceeding CHI '03 extended abstracts on Human factors in computing systems (pp. 964-965). Florida: ACM.

Sheikh, J. I., y Yesavage, J. A. (1986). Geriatric Depression Scale (GDs): Recent evidence and development of a shorter version. Clinical Gerontologist: The Journal of Aging and Mental Health, 5(1-2), 165-173. doi:10.1300/J018v05n01_09

Spielberger, C. D., Gorsuch, R. L., y Lushene, R. E. (1970). sTAI, Manual for the state-trait anxiety inventory. California: Consulting Psychologist Press.

Wellman, B., y Frank, K. (2001). Network capital multi-level world: Getting support from personal communities. En N. Lin, K. Cook, \& R. Burn (Eds.). Social capital: Theory and research (pp. 233-273). Hawthorne, NY: Aldine de Gruyter.

Van de Watering, M. (2005). The Impact of Computer Technology on the Elderly. Recuperado de http://collab.ist.psu. edu/future-fall2008/team-space/life-long-engagement/life-long-engagment-files/ HCI_Essay_Marek_van_de_Watering.pdf 